\title{
Mejora continua en el servicio que reciben los usuarios, Dirección Regional Agraria Amazonas, Perú, 2017
}

\section{Continuous improvement in the service received by users, Amazonas Regional Agrarian Directorate, Peru, 2017}

\author{
Benjamín Roldan Polo Escobar ${ }^{1}$, Rosa Ysabel Bazán Valque², Alex Javier Sánchez Pantaleón ${ }^{3}$
}

\section{RESUMEN}

La investigación tuvo como objetivo determinar el nivel de mejora continua, en los procesos de servicios que reciben los usuarios de la Dirección regional Agraria Amazonas aplicando el "Modelo de las Esferas concéntricas de Kaizen". La población de estudio estuvo constituida por los trabajadores de la institución, compuesta por (11) directores de línea, (49) profesionales, (60) técnicos. La muestra estuvo representada por (120) trabajadores, se utilizó un muestreo por conveniencia. Para la recolección de datos se utilizó una encuesta: pre tes y post test. Se encontró respecto al nivel de liderazgo en los trabajadores que el $47 \%$ tiene un avance medio y el $12 \%$ tiene un avance parcial. En el nivel de desarrollo de las personas el $44 \%$ tienen un avance medio y el $6 \%$ tiene un avance inicial. En el nivel de interés por el usuario externo el $45 \%$ tiene un avance medio y el $14.0 \%$ tiene un avance parcial. En la mejora de la competitividad tienen un avance medio del $37 \%$ y el $8 \%$ en un avance inicial y el nivel de gestión y mejora de los procesos el $49 \%$ tiene un avance medio y un avance inicial del $5 \%$. Se concluye que el modelo influye positivamente en los niveles de la Calidad de los Servicios que perciben los usuarios de la Dirección regional agraria de la ciudad de Chachapoyas, Perú.

Palabras clave: Mejora continua, Kaizen, procesos.

\section{ABSTRACT}

The objective of the research was to determine the level of continuous improvement in the service processes that users of the Amazonas Agrarian Regional Directorate receive by applying the "Kaizen Concentric Spheres Model". The study population was constituted by the workers of the institution, composed of (11) line directors, (49) professionals, (60) technicians. The sample was represented by (120) workers from the Regional Agrarian Directorate, a convenience sampling was used. For data collection, a survey was used: pre-test and post-test. Regarding the level of leadership in the workers, $47 \%$ have a medium advance and $12 \%$ have a partial advance. At the level of development of people, $44 \%$ have an average advance and $6 \%$ have an initial advance. Regarding the level of interest for the external user, $45 \%$ have a medium advance and $14.0 \%$ have a partial advance. In improving competitiveness, they have an average advance of $37 \%$ and $8 \%$ in an initial advance and the level of management and improvement of processes $49 \%$ have an average advance and an initial advance of $5 \%$. It is concluded that the model positively influences the levels of the Quality of Services perceived by users of the Regional Agrarian Directorate of the city of Chachapoyas, Peru.

Keywords: Continuous improvement, Kaizen, processes.

\footnotetext{
Doctor en Gestión Pública y Gobernabilidad - Docente en la Facultad de Ciencias Económicas y Administrativas perteneciente a la Escuela Profesional de Administración de Empresas de la Universidad Nacional Toribio Rodríguez de Mendoza de Amazonas. Correo electrónico: benjamin.polo@gmail.com

${ }^{2}$ Doctora en Gestión Pública y Gobernabilidad - Docente en la Facultad de Ciencias Económicas y Administrativas perteneciente a la Escuela Profesional de Economía de la Universidad Nacional Toribio Rodríguez de Mendoza de Amazonas. Correo electrónico: rosa.bazan@untrm.edu.pe

${ }^{3}$ Maestro en Gestión Pública - Docente en la Facultad de Ciencias Económicas y Administrativas perteneciente a la Escuela Profesional de Economía de la Universidad Nacional Toribio Rodríguez de Mendoza de Amazonas. Correo electrónico: alex.sanchez@untrm.edu.pe
} 


\section{INTRODUCIÓN}

En la actualidad los directivos de las diferentes organizaciones publica y privadas, están preocupados por implementar procedimientos con aspectos incrementales, que le permitan una mejora de la calidad de cada uno de los procesos en el desarrollo institucional y presentarse como modelo a otros organismos públicos, a partir de estrategias innovadoras para todas las organizaciones ligadas a la mejora en la atención de los usuarios, actores ligados al sector agrario y convertirlas en puntos clave de poder realzar los diferentes aspectos comerciales en toda su cadena productiva.

Los aspectos de innovación de una institución a partir de la mejora continua que le permita optimar la competitividad de la organización se sustentadas en el "Modelo de las Esferas concéntricas de Kaizen" con el propósito de desarrollar actividades que permitan encontrar la satisfacción de sus usuarios y de los trabajadores.

cuyo impacto se verá en los usuarios tendrá

El presente trabajo de investigación partió de un diagnóstico el cual ha permitido revelar la identificación de posibles falencias y en consecuencia proponer alternativas de mejorar su competitividad en sus diferentes áreas, la cual se viabilice mediante la productividad en una manera permanente y que sea sostenible en el tiempo, cuyo impacto se verá reflejado en los usuarios, actores locales quienes constituyen un número bastante significativo en la región, con una comunicación abierta y con un sistema de información trabajando de una forma más ágil, oportuna, eficaz y convincente.

A partir de ello se resalta a "Kaizen, como un análisis teórico de la mejora continua" (Imai,1986), asimismo como el "Mejoramiento continuo que involucra a todos, gerente y trabajadores por igual" (Imai M., 1989). Para Newitt D. (1996), la definición de Imai (1986, 1989) se basa en que: "la palabra Kaizen es una derivación de dos ideogramas japonesas que significan: $\mathrm{KAI}=$ Cambio, ZEN = Bueno (para mejorar)" (Farley, 1999); (Newitt D., 1996). Es decir: "Mejora Continua o Principio de Mejora Continua" (Lillrank, 1995); (Kano, 1989).

De esta manera: "la primera óptica de la definición del Kaizen se basa entonces en sustentar su presencia, como un elemento organizacional en la que la participación de los empleados impacta directamente en la mejora de los procesos de trabajo" (Senge, 1990); (Elgar \& Smith, 1994). En este sentido, Bessant (2003) indica que: "la movilización y la participación de los empleados, genera un canal o un medio para que los mismos, puedan contribuir al desarrollo de la compañía. En términos sencillos, trabajando con las manos, pero utilizando el cerebro para pensar" (Bessant, 2003). Así mismo: "Esta idea, se compara y es similar a los estudios iniciales de la Escuela de las Relaciones Humanas, en las que Mayo, Maslow, McGregor y Herzberg argumentaban dicha aproximación del Management" (Malloch, 1997).

De hecho, en ocasiones "el Kaizen ha sido visto como una fuerza ética (interna que se encuentra en uno mismo) de cada trabajador, que es capaz de resolver problemas en el día a día, plenamente convencido y de manera voluntaria" (Styrhe, 2001).

"Por ello ciertos autores, comienzan a verlo como una filosofía de vida, en la que se pueden abarcar los ámbitos personales, familiares, sociales y por supuesto la vertiente del trabajo" (Imai, 1986).

"El Kaizen significa Mejoramiento continuo, pero mejoramiento todos los días, a cada momento, realizado por todos los empleados de la organización, en cualquier lugar de la empresa. Y que va de pequeñas mejoras incrementales a innovaciones drásticas y radicales”(Imai, 2006) y (Imai, 2007).

"Una filosofía de gestión que genera cambios o pequeñas mejoras incrementales en el método de trabajo (o procesos de trabajo) que permite reducir despilfarros y por consecuencia mejorar el rendimiento del trabajo, llevando a la organización a una espiral de innovación incremental" (SuárezBarraza, 2007).

Finalmente, también se encuentra la línea de autores que indican que: "el Kaizen o la Mejora Continua en su caso, puede ser entendido, como un elemento más de la Gestión por Calidad Total" (Crosby, 1979); (Ishikawa, 1986); (Deming, 1986), o "como la plataforma básica de Sistema de Producción Toyota o el Pensamiento Esbelto (o Lean Thinking) caracterizado por la participación de los empleados en la solución de los problemas o desperdicios (Muda) que surgen en el trabajo cotidiano; la forma en que se ejecuta dicha eliminación es a través de equipos de mejora o de la aplicación de las 5'S y la estandarización" (Womack, Jones, \& D. Toss, 1990).

Para Ohno (1978), el fundador del Sistema de Producción Toyota la mejora continua o Kaizen se entendía como que "la mejora tiene dos vertientes principales: es eterna e infinita"

Esto reafirma que: “el Kaizen no es un programa de mejora continua aislado, sino que por el contrario es considerado en dicha organización como un elemento filosófico de su modelo de negocio-el 
Toyota Way 2001”.

Según el planteamiento de Cheser (1994), argumenta que: “el Kaizen va más allá de la forma con la que se define en occidente, es decir, de la Mejora Continua en otros.

"Kaizen desde su orientación occidental como término de Mejora Continua es más abundante que la de su orientación japonesa, sigue siendo señalada por algunos autores como (2005).

Dentro de esta orientación más occidental, el Kaizen es abordado desde: "un ángulo gerencial y organizacional más práctico, delimitando al término mismo, en forma de metodología y/o técnica conformada por conjunto de herramientas necesarias para eliminar las actividades que no agregan valor a los procesos de trabajo, los llamados mudas en japonés. Entre ellas están el Kaizen Blitz (Cuscela, 1998).

Esfera concéntrica del tipo 1 - el Kaizen como filosofía gerencial: "Involucra una serie de principios y valores que soportan al sistema de gestión de la organización: prácticas, herramientas de gestión, consideradas en su origen japonés, dentro de las cuales se incluyen la Gestión por Calidad Total de toda la Empresa" (Imai, 1989)

Esfera concéntrica del tipo 2: el Kaizen como "Un elemento más de la Gestión por Calidad Total (TQM)". La literatura indica que las raíces de los programas de mejora en occidente se pueden trazar hasta los trabajos del gobierno norteamericano en el llamado Training Within Industry durante la Segunda Guerra Mundial; este programa incluyó el entrenamiento y educación de los supervisores con técnicas de control estadístico y mejora continua de los procesos" (Schroeder y Robinson, 1991).

La Mejora Continua puede ser vista como una capacidad organizacional, ya sea formando parte del TQM o como otro tipo de programa de mejora o innovación (Bessant y Caffyn 1997; Bessant 2003).

Esfera concéntrica del tipo 3 - El Kaizen como principio teórico de metodologías y técnicas de mejora: esta perspectiva se fundamenta en: “El enfoque al corto plazo del mismo. Trata, por ende, de eliminar mudas en eventos Kaizen intensivos (sólo algunos días), en los cuales se utilizan las ideas de mejora de los empleados que conocen los procesos de trabajo a fondo" (Cuscela, 1998).

“Las tres esferas concéntricas propuestas representan una primera aproximación por intentar entender al Kaizen, por lo que es evidente, que esta forma de agrupación realizada a través de un análisis teórico puede tomar diferentes formas de plantearlas dependiendo del ángulo con que se aborde" (SuárezBarraza \& Miguel-Dávila, 2008).

La Mejora continua "está relacionado en la vida personal, familiar, social y de trabajo. Cuando se aplica al lugar de trabajo como un mejoramiento continuo progresivo involucra a todos gerentes y trabajadores por igual" (Imai, 1989).

Así mismo, “el Kaizen es definido como una filosofía que requiere que todas las personas, todos los días, en todos los lugares puedan y deban mejorar" (Imai, 2006).

Además, "esta mejora continua, constante e incremental puede abarcar los ámbitos personales, familiares, sociales y por supuesto del trabajo" (Imai, 1989); (Malloch, 1997). En este sentido, "su práctica les permite a las personas trabajar y vivir bajo un contexto de respeto, ética, honor y armonía por el entorno que le rodea" (Styrhe, 2001).

La Mejora Continua de Procesos (MCP) bajo la filosofía Kaizen: "Un proceso de toda la organización que se enfoca en un continuo e incremental esfuerzo de innovación (Bessant y Caffyn, 1997).

Según Jorgensen (2003), indica que la Mejora continua es: "Un proceso planeado, sistemático y organizado de toda la organización que busca un cambio incremental de las prácticas existentes que redunde en el rendimiento de la compañía" (Jorgensen, Boer, \& Gertsen, 2003).

La justificación de la investigación parte de una propuesta de utilidad que se sustenta científicamente con teorías relacionadas al Kaizen. Según Montabon (2005) indica que: "ha explorado en las áreas de servicio la aplicación del Kaizen de la tercera perspectiva del Kaizen, lo que comienza a enriquecer las investigaciones haciendo esta distinción entre manufactura y servicio" (Montabon, 2005).

Además, en el enfoque práctico, la distinción de las instituciones innovadoras se encuentra cada vez más con la oportunidad de constituir a todo el recurso humano en sus planes organizacionales, considerando sus objetivos y actividades con características propias. La importancia de proponer un modelo a partir de las tres esferas concéntricas del Kaizen permite realizar un esbozo con herramientas sustentadas dentro de la Gestión Estratégica, atención al público, la generación de Información, este proceso radica en que los resultados que se obtengan nos van a exponer una mejor toma de decisiones, al concluir se espera que el actual trabajo contribuirá de manera significativa a mejorar y e integrar diversos aspectos como las necesidades de los usuarios, flujos de información permitan a los decisores una 
adecuada toma de decisiones.

Dentro del aspecto Metodológico. según Ulmer (1987) y Hopkins (1983), indican que "Están comenzando a comprender que los trabajadores no se retienen únicamente por el salario, el descubrimiento de los factores que influyen en su grado de satisfacción empieza a ser un elemento de interés. Por ello, el análisis de la satisfacción laboral en las empresas debe de realizarse por las siguientes razones"

Y en el aspecto Legal, Se fundamenta dentro de la Ley Marco de Modernización de la Gestión del Estado (2002) que busca: "obtener mayores niveles de eficiencia del aparato estatal, de manera que se logre una mejor atención a la ciudadanía, priorizando y optimizando el uso de los recursos públicos, teniendo como objetivo, entre otras poner al Estado al servicio de la ciudadanía, con canales efectivos de participación ciudadana, descentralizado y desconcentrado, transparente en su gestión y con servidores públicos calificados y adecuadamente remunerados" (Ley Marco de Modernización de la Gestión del Estado, 2002).

El articulo busca generar el conocimiento a partir de un planteamiento estratégico que nos permita alcanzar el éxito el cual se hace necesario en el día a día dentro del campo empresarial, para lo cual se planteó como objetivo general determinar el nivel de mejora continua, en los procesos de servicios que reciben los usuarios de la Dirección regional Agraria Amazonas aplicando el "Modelo de las Esferas concéntricas de Kaizen". Que permita la optimización de procesos, permanentes cambios, la generación de nuevos conocimientos, la inserción de nueva tecnología hace tener un nuevo giro dentro del entorno institucional obligando a los colaboradores a perfeccionarse y mejorar sus lineamientos de política, así como adquirir una nueva cultura organizacional y herramientas que les permitan desarrollarse.

\section{MATERIAL Y MÉTODO}

Diseño: La presente investigación está enmarca dentro de un aspecto cuantitativo es de tipo descriptivo, analítico y propositivo en tanto se propuso un modelo, a partir de la técnica de campo que permitió el contacto directo con la población objetivo, y el acopio de testimonios que permitieron confrontar la teoría con la práctica en la búsqueda de la verdad objetiva. Población muestral: La población muestral de estudio estuvo constituida por los trabajadores de la Dirección regional agraria Amazonas, compuesta por (11) directores de línea,
(49) profesionales, (60) técnicos.

Relación de trabajadores de la Dirección regional agraria Amazonas.

\begin{tabular}{|c|c|c|c|c|c|}
\hline Tipo de pruebas & $\begin{array}{c}\text { Nro. } \\
\text { Trabajadores }\end{array}$ & 5 & $\begin{array}{r}\text { Cén } \\
\text { Mascuino }\end{array}$ & $\begin{array}{l}\text { nero } \\
\text { Femenino }\end{array}$ & Total \\
\hline Direcoiones de ínea & 11 & 9.17 & 5 & 8 & 11 \\
\hline Profesionales & 49 & 4083 & 30 & 19 & 49 \\
\hline Téconicos & 45 & 37.50 & 30 & 15 & 45 \\
\hline Personal de Apojo & 15 & 1250 & 10 & 5 & 15 \\
\hline Total & 120 & 100 & 75 & 45 & 120 \\
\hline
\end{tabular}

Muestreo: se utilizó un muestreo por conveniencia. Para la recolección de datos se utilizó una encuesta: pre tes y post test.

\section{Construcción del instrumento de mejora continua:}

Como parte de la solución a un problema de tipo práctico se construyó un modelo basado en las esferas concéntricas de Kaizen, nace en un área particular del conocimiento, a partir de un diagnóstico se puntualizó e identifico las necesidades, generando procesos interpretativos a partir de sus objetivos específicos a) diagnóstico de las principales características con un enfoque en determinar el nivel de liderazgo. b) Aplicación del modelo de mejora continúa sustentado en las esferas concéntricas de Kaizen que permiten el fortalecimiento de capacidades y desarrollo de las personas. c) Evaluar la mejora continua en el interés del usuario externo d). evaluar la gestión y mejora de los procesos.

Respecto a medir el nivel de la mejora continua, en los procesos de servicios que reciben los usuarios de la Dirección regional Agraria Amazonas sustentado en el "Modelo de las Esferas concéntricas de Kaizen" consistió 1) Identificar los procesos de servicios que reciben los usuarios de la Dirección regional Agraria Amazonas. 2) Generar las directrices futuras en sus diferentes áreas, es decir, plantear perspectivas de mejorar en base a los resultados de un proceso de investigación particular.3) propiciar los cambios tecnológicos y administrativos que vayan paralelos a la modernización, que le permita mejorar los procesos y servicios, a partir del cambio de la actitud general de la institución. El modelo fue validado a criterio de juicios de expertos.

El Diseño se refiere al "plan o estrategia concebida para obtener la información que se desea" (Hernández, Fernández, \& Baptista, 2006) 
La investigación está fundamentada como una propuesta teórica de solución al problema planteado, en un proceso metódico a partir de un diagnóstico contemplando pasos determinantes como la descripción, el análisis, la comparación, la explicación y la predicción, en ese sentido la evaluación tuvo un Antes y un Después de la aplicación del modelo de mejora continua en los procesos de servicios que reciben los usuarios de la Dirección regional Agraria Amazonas sustentado en el "Modelo de las Esferas concéntricas de Kaizen" aplicándose una encuesta de 18 preguntas, la misma que fue sometida a juicio de expertos y confiabilidad según coeficiente Alfa de Cronbach (0.77). Para el análisis de datos se utilizó el software estadístico SPSS versión 25.

\section{RESULTADOS}

Tabla 1. Nivel porcentual de liderazgo.

\begin{tabular}{lcc}
\hline Nivel de liderazgo & $\begin{array}{c}\text { Porcentaje } \\
\text { Pre test }\end{array}$ & $\begin{array}{c}\text { Porcentaje } \\
\text { post test }\end{array}$ \\
\hline Estado Inicial & $20 \%$ & $0 \%$ \\
Avance Parcial & $25 \%$ & $12 \%$ \\
Avance medio & $40 \%$ & $47 \%$ \\
Avance significativo & $15 \%$ & $28 \%$ \\
Avance destacable & $0 \%$ & $13 \%$ \\
\hline \multicolumn{1}{c}{ Total } & $100 \%$ & $100 \%$ \\
\hline
\end{tabular}

Tabla 2. Nivel porcentual de desarrollo de las personas.

\begin{tabular}{lcc}
\hline $\begin{array}{c}\text { Nivel desarrollo de las } \\
\text { personas }\end{array}$ & $\begin{array}{c}\text { Porcentaje } \\
\text { Pre test }\end{array}$ & $\begin{array}{c}\text { Porcentaje } \\
\text { post test }\end{array}$ \\
\hline Estado Inicial & $12 \%$ & $6 \%$ \\
Avance Parcial & $30 \%$ & $20 \%$ \\
Avance medio & $40 \%$ & $44 \%$ \\
Avance significativo & $18 \%$ & $23 \%$ \\
Avance destacable & $0 \%$ & $7 \%$ \\
\hline \multicolumn{1}{c}{ Total } & $100 \%$ & $100 \%$ \\
\hline
\end{tabular}

Tabla 3. Nivel porcentual de interés por el usuario externo.

\begin{tabular}{lcc}
\hline $\begin{array}{c}\text { Nivel interés por el } \\
\text { usuario externo }\end{array}$ & $\begin{array}{c}\text { Porcentaje } \\
\text { Pre test }\end{array}$ & $\begin{array}{c}\text { Porcentaje } \\
\text { post test }\end{array}$ \\
\hline Estado Inicial & $10 \%$ & $0 \%$ \\
Avance Parcial & $35 \%$ & $9 \%$ \\
Avance medio & $45 \%$ & $50 \%$ \\
Avance significativo & $10 \%$ & $25 \%$ \\
Avance destacable & $0 \%$ & $16 \%$ \\
\hline \multicolumn{1}{c}{ Total } & $100 \%$ & $100 \%$ \\
\hline
\end{tabular}

Tabla 4. Nivel porcentual de resultados en la mejora de la competitividad.

\begin{tabular}{lcc}
\hline $\begin{array}{c}\text { Nivel resultados en la } \\
\text { mejora de la } \\
\text { competitividad }\end{array}$ & $\begin{array}{c}\text { Porcentaje } \\
\text { Pre test }\end{array}$ & $\begin{array}{c}\text { Porcentaje } \\
\text { post test }\end{array}$ \\
\hline Estado Inicial & $25 \%$ & $8 \%$ \\
Avance Parcial & $40 \%$ & $30 \%$ \\
Avance medio & $25 \%$ & $37 \%$ \\
Avance significativo & $5 \%$ & $15 \%$ \\
Avance destacable & $5 \%$ & $10 \%$ \\
\hline \multicolumn{1}{c}{ Total } & $100 \%$ & $100 \%$ \\
\hline
\end{tabular}

Tabla 5. Nivel porcentual de resultados en la mejora de la competitividad.

\begin{tabular}{lcc}
\hline $\begin{array}{c}\text { Nivel Gestión y } \\
\text { mejora de los } \\
\text { procesos }\end{array}$ & $\begin{array}{c}\text { Porcentaje } \\
\text { Pre test }\end{array}$ & $\begin{array}{c}\text { Porcentaje } \\
\text { post test }\end{array}$ \\
\hline Estado Inicial & $28 \%$ & $5 \%$ \\
Avance Parcial & $37 \%$ & $18 \%$ \\
Avance medio & $30 \%$ & $49 \%$ \\
Avance significativo & $5 \%$ & $21 \%$ \\
Avance destacable & $0 \%$ & $7 \%$ \\
\hline \multicolumn{1}{c}{ Total } & $100 \%$ & $100 \%$ \\
\hline
\end{tabular}

\section{DISCUSIÓN}

En la actualidad cualquier ensayo que busque determinar el nivel de mejora continua en los procesos de servicios que reciben los usuarios de la Dirección regional Agraria Amazonas, a partir del "Modelo de las Esferas concéntricas de Kaizen", se tiene que dicha técnica de gestión administrativa debe partir de un diagnóstico situacional, como mejora en la institución, y tener en cuenta los supuestos teóricos que permite su aplicación y formalización dentro de un marco de gestión por procesos. Abriendo así, una nueva línea para futuros trabajos científicos.

Respecto a la evaluación de la información Nivel de liderazgo, (tabla 1), se puede observar en el pre test que la mayoría de encuestados respecto al nivel de liderazgo el (40\%) se encuentra en un nivel avance medio limitando las tomas de decisiones en las actividades que se realizan en la institución y que relacionado al tema de mejora continua (post test) se puede observar que el (12\%) se encuentra en un nivel parcial y un $(47 \%)$ se encuentra con un avance medio lo que demuestra que a partir de los lineamientos impartidos existe un proceso de mejora.

En ese sentido Según el enfoque de "Kaizen, al contrario de otras filosofías empresariales, indica no se trata de realizar grandes cambios, más bien se 
enfoca en realizar mejoras pequeñas pero continuadas en todas las actividades, es una cuestión paso a paso y no de grandes revolcones" (Maslow, 2001).

Asimismo "el Kaizen genera pensamientos orientados al proceso, pues este debe ser mejorado, para obtener mejores resultados"; Además, indica que está “orientado a las personas, dirigiéndose a los esfuerzos de las mismas. No solo se valoran los resultados obtenidos, sino también la habilidad, el esfuerzo y el espíritu de superación demostrados" (Gil \& Giner, 2010).

En relación a la evaluación de la información Nivel desarrollo de las personas, (tabla 2), se puede observar en el pre test que $(40 \%)$ de encuestados demuestran un nivel de desarrollo del con un nivel avance medio limitando algunas responsabilidades y/o funciones que permita un avance de las actividades que se realizan en la institución y que relacionado al tema de mejora continua (post test) se puede observar que el (6\%) todavía se encuentra en un nivel inicial asimismo el (44\%) se encuentra en un nivel de avance medio demostrando una preocupación por mejorar su formación a partir de capacitaciones, además se puede apreciar un avance significativo del (23\%) lo que demuestra que a partir de los interacciones impartidas relacionadas al proceso de mejora continua, existe una perspectiva de mejorar en la actividad que realiza.

El aspecto organizacional y la estimulación permanente del personal. Según estudios de investigación indican que no es solamente un supuesto ideológico, sino que debe estar reflejado en la actitud de los trabajadores y debe ser persistente en el tiempo la aplicación e implementación de las operaciones de perfeccionamiento, con todas las capacidades enmarcadas será un sostén de la gerencia y de los colaboradores de la institución intervenida, las mismas que deben estar acondicionadas a las situaciones delimitadas de cada institución la cual permita en un corto y/o mediano plazo la mejora esperada.

En relación a la evaluación de la información Nivel de interés por el usuario externo, (tabla 3 ), se puede observar en el pre test que el (45\%) de encuestados demuestran un nivel de interés de avance medio por el usuario externo limitando la comunicación y las interacciones con el productor que permita una atención esmerada por parte de la institución y que relacionado al tema de mejora continua (post test) se puede observar que existe un $(9 \%)$ con un nivel de avance parcial adicionalmente se encuentra una mejora relativa, del (50\%) localizado en un nivel de avance medio lo cual se ve que existe una preocupación por mejorar su atención al cliente a partir de capacitaciones, además en este nivel se puede apreciar un avance significativo del (25\%) lo que demuestra que a partir de las mismas interacciones impartidos relacionadas al proceso de mejora continua, existe una perspectiva de mejorar en la atención al usuario externo.

En el presente estudio se hace referencia a la Teoría Kaizen, la cual establece que: "el cambio continuo que efectúa la empresa para mejorar y lograr la calidad total en sus procesos. Este enfoque gerencial japonés, cuyo proverbio es: Hoy mejor que ayer, mañana mejor que hoy, involucra a todos los miembros de la organización, con una visión holística a fin de solventar los inconvenientes y renovar sus formas de trabajo constantemente" (Imai, 2006).

En relación a la evaluación de la información Nivel de resultados en la mejora de la competitividad, (tabla $4)$, se puede observar en el pre test que el $(40 \%)$ de encuestados demuestran un nivel de interés de avance parcial en los resultados en la mejora de la competitividad limitando el avance de las tareas encomendadas e indicadores lo que limita el avance de las principales acciones de la institución y que relacionado al tema de mejora continua (post test) se puede observar que existe un $(8 \%)$ con un nivel de estado inicial lo que demuestra un aspecto pasivo frente a la competitividad, a pesar de ello se encuentra una mejora relativa, con el $(37 \%)$ demostrando un avance medio en este proceso, existiendo una preocupación por mejorar dichos resultados a partir de motivaciones, acciones y capacitaciones, además en este nivel se puede apreciar un avance significativo del (15\%) dentro de un avance significativo lo que demuestra que a partir de las mismas acciones y/o lineamientos impartidos relacionadas al proceso de mejora continua, existe una perspectiva de mejorar en las principales actividades encomendadas.

Los Pilares del Kaizen que fundamentan. Esta base filosófica hace que la mejora continua se convierta en una cultura para ser mejores que va más allá de lo económico y en este sentido es casi una cuestión ética que se entremezcla con las veteranas teorías de Maslow" (Maslow, 2001).

Para la evaluación se integró a un equipo multidisciplinario conformado por especialistas, ingenieros y técnicos. Según el objetivo del presente modelo se facilitó de todas las herramientas y conocimientos sobre el tema con explicaciones muy sencillas, con la finalidad de empoderar de las nuevas estrategias del Evento Kaizen con la finalidad de que el personal en un tiempo relativamente corto, esté predispuesto al cambio. Simultáneamente a ello se hizo participar al personal con sus opiniones de perfeccionamiento sobre el objetivo propuesto, se exploran las opiniones de todos los colaboradores buscando una mejora sustancial en todas las áreas, 
para lo cual se generará un plan de trabajo y se trabaja en las mejoras.

En relación a la evaluación de la información Nivel de gestión y mejora de los procesos, (tabla 5), se puede observar en el pre test que el $(37 \%)$ de encuestados demuestran un nivel de interés parcial en la gestión y mejora de los procesos limitando el avance de la gestión la misma que es evaluada con indicadores de las principales acciones de la institución y que relacionado al tema de mejora continua (post test) se puede observar que existe un (5\%) de los encuestados se encuentra en el estado inicial y un $(49 \%)$ se está en un nivel medio encontrando una mejora sustancial, en este proceso existiendo una preocupación por conseguir resultados a partir de las motivaciones e incentivos, y que a partir de ello se puede apreciar avances significativos en los procesos de intervención relacionadas a la mejora continua, en las actividades previstas.

Según Yonque, García, y Ráez (2002) reiteran que: "el Kaizen es una forma de vida, que involucra a gerentes y trabajadores en ese proceso de mejora progresiva de las organizaciones". En este sentido, el "mejoramiento continuo orientado a las personas, privilegia sus esfuerzos como seres humanos, miembros de una familia, de una sociedad, organización, lo cual trasciende a todos los cargos, independientemente de si se trata de la alta dirección o el nivel operativo".

Según Montabon (2005) afirma que: "también ha explorado en las áreas de servicio la aplicación del Kaizen de la tercera perspectiva del Kaizen, lo que comienza a enriquecer las investigaciones haciendo esta distinción entre manufactura y servicio". En esa misma línea también: "se puede abrir una línea de investigación acerca de la investigación del Kaizen en el sector público, que algunos autores han comenzado a explorar" (Suárez-Barraza y RamisPujol, 2008); (Imai M, 2007)

"Mediante la filosofía Kaizen, se preocupan por llevar las mejoras a su puesto de trabajo. por ejemplo, buscará maneras más eficientes de hacerlo y no solo se preocupará de hacerlo de forma mecánica. Se basa en algunos principios como son: Disciplina, círculos de calidad, sistema de sugerencias, Seiketsu ( 1 impieza), Seiton y Seiri (orden)" (www.pymesyautonomos.com, 2010).

\section{CONCLUSIONES}

1. Respecto al nivel de liderazgo en los trabajadores el $47 \%$ tiene un avance medio y el $12 \%$ tiene un avance parcial.

2. En el nivel de desarrollo de las personas el $44 \%$ tienen un avance medio y el $6 \%$ tiene un avance inicial.

3. En el nivel de interés por el usuario externo el $50 \%$ tiene un avance medio y el $9 \%$ tiene un avance parcial.

4.En el nivel de los resultados en la mejora de la competitividad tienen un avance medio del $37 \%$ y el $8 \%$ en un avance inicial y

5. En el nivel de gestión y mejora de los procesos el $49 \%$ tiene un avance medio y un avance inicial del $5 \%$.

\section{REFERENCIAS BIBLIOGRÁFICAS}

Association, Japan Human Relations. (1990). Kaizen Teian. Cambridge: Productivity Press. www.slideshare.net

Barrera, M. (2007). Modelos epistémicos en educación e investigación. Venezuela: Ediciones Quirón. Publicaciones.urbe.edu

Bessant, J. (2003). High Involvement Innovation. Chichester West Sucess England: John W i l e y a n d S o n g s L t d. p u b l i c a c i o n e s. u r b e e d u : www.slideshare.net

Bessant, J. y Caffyn, S. (1997). High-Involvement Innovation Through Continuous Improvement. International Journal Technology Management, 7-28. www.unileon.es, www.slideshare.net

Bhuiyan, N. A. (2005). An Overview of Continuous Improvement: From the Past to the Present, Management Decision. 43, No. 5, pp. 761771 .

Cheser, R. (1994). Kaizen is More Than Continuous Improvement. Quality Progress, 23-25. www.slideshare.net

Crosby, P. (1979). Quality is Free. New York: McGraw-Hill. www.tdx.cat

Cuscela. (1998). Kaizen Blitz: Attacks Work Processes at Dana Corp. IIEE Solutions, 2931. Universidad de Costa Rica

Deming, W. E. (1986). Out of the crisis. Cambridge, Massachusetts: MIT/CAES.

Elgar, T., \& Smith, C. (1994). Global Japanization: The Transnational. Transformation for the Labour Process. London: Routledge.

Farley, C. (1999). "Despliegue de Políticas del KAIZEN". XI Congreso de Calidad Total 
organizado por el Centro de Productividad de Monterrey, Nuevo León México. Fundación Mexicana de la Calidad Total y Centro de Productividad de Monterrey. Monterrey Nuevo León México: Fundación Mexicana de la Calidad Total y Centro de Productividad de Monterrey.

Galeano, M. (2004). Diseño de proyectos en la investigación cualitativa. Colombia: Fondo Editorial Universidad EAFIT. Publicaciones.urbe.edu

Gil, M., \& Giner, F. (2010). Cómo crear y hacer funcionar una empresa. España: Ediciones ESIC. Obtenido de publicaciones.urbe.edu

Hernández, Fernández, \& Baptista. (2006). Metodología de la Investigación. www.wordpress.com

Hopkins, A. H. (1983). Work and Job Satisfaction in the Public Sector. Totowa: Rowman \& Allanheld.

Imai, M. (1986). Kaizen-The key to Japan's Competitive Success. New York: Random House. publicaciones.urbe.edu: www.slideshare.net

Imai, M. (1989). Kaizen, la clave de la ventaja competitiva japonesa. México D.F.: CECSA. www.tdx.cat: bibadm.ucla.edu.ve

Imai, M. (2006). What is total Flow management under Kaizen focus? Three days Conference lecturer. España. publicaciones.urbe.edu: www.slideshare.net

Imai, M. (2007). Mejorar la calidad es la mejor forma de reducir los costes. Diario de Negocios la Gaceta, 36. www.tdx.cat, w w w.revis tas.un a 1 . ed u. co; www.slideshare.net; www.gestiopolis.com

Ishikawa, k. (1986). What is Total Quality Control? The Japanese Way. Englewood Cliffs. NJ: Prentice Hall. www.tdx.cat

Jorgensen, F., Boer, H., \& Gertsen, F. (2003). Jumpstarting Continuous Improvement Through self-assessment. International Journal of Operations \& Production Management, 1260-1278. www.slideshare.net

Kano, N. (1989). Continuous Improvement-Quality Control Circles in Japanese Industry. Michigan: Center for Japanese Studies.

Lillrank, P. (1995). The transfer of Management
Innovations from Japan. Organization Studies (Vols. 16, No. 6, pp. 971-989).

Ley Marco de Modernización de la Gestión del Estado. ( $29 \mathrm{de} 01 \mathrm{de} 2002)$. www.inicam.org.pe. Ley $\mathrm{N}^{0} 27658$. w w w . i n i c a m o r g p e, www.inicam.org.pe/normativa/legislacion/L ey27658.pdf

Malloch, H. (1997). Strategic and HRM aspects of kaizen: a case study. New Technology, Work and Employment, New Technology, Work and Employment, 12, No. 2, pp. 108-122., 108-122.

Maslow (2001). Teoría filosófica de la mejora continua. www.gestiopolis.com, 2001

Montabon, F. (2005). Using Kaizen Events for Back Office Processes: Recruitment of frontline Supervisor Co-ops", Total Quality Management and Business Excellence. 16(10), 1139-1147. Kaizen: Un análisis teórico de la Mejora Continua: www.tdx.cat, w w w . s l i d e s h a r e. n e t ; https://dialnet.unirioja.es/descarga/articulo/3 117757.pdf

Newitt, D. (1996). Beyond BPR \& TQM - Managing through Processes: Is Kaizen Enough?. Industrial Engineering Conference Proceeding. London: Institution of Electric Engineers. docplayer.es

Ohno, T. (1978). Toyota Production System. Beyond Large-Scale Production. New York, NY: Productivity Press. www.slideshare.net

Schroeder D. y Robinson, A. (1991). America's Most Successful Export to Japan-Continuous Improvement Programmes. Sloan Management Review, 67-81. www.slideshare.net

Senge, P. (1990). The Fifth Discipline. New York. N.Y: Double Day.

Suárez-Barraza M.F. (2007). Esferas concéntricas Kaisen. Rrevistas.uexternado.edu.com

Suárez-Barraza, M. F., \& Miguel-Dávila, J.-Á. (2008). dialnet.unirioja.es. Encontrando al Kaizen. Un análisis teórico de la mejora $\begin{array}{lllllllll}c & o & n & t & i & n & u & a\end{array}$. https://dialnet.unirioja.es/descarga/articulo/3 117757.pdf

Suárez-Barraza y Ramis-Pujol. (2008). Perspectivas del Kaizen. www.slideshare.net 
Styrhe, A. (2001). Kaizen, Ethics, and Care of the Operations: Management after Empowerment. Journal of Management Studies, 38, No. 6, pp. 795-810., 795-810.

Ulmer, D. L. (1987). Job Satisfaction of Community Hospital Educators. Dissertation Abstracts International. (49). redalyc.uaemex.mx

Womack, J. P., D.T. Jones, \& D. Toss. (1990). The Machine that Changed the World. New York, N.Y.: R aw s o n, A s s o c i a t e s. www.doc.isiri.org.ir

Yonque, J., García, M., \& Ráez, L. (2002). sisbib.unmsm.edu.pe. Kaizen o la mejora continua. Industrial Data (C) UNMSM. http://sisbib.unmsm.edu.pe/bibvirtual/publi caciones/indata/v05_n1/kaisen.htm 